\title{
The causes of obesity: an in-depth review
}

\begin{abstract}
The global sharp rise in the prevalence of obesity has made it a critical public health issue in the last few decades. The obesity epidemic deploys a detrimental impact on the economy with its high healthcare cost. Therefore, Obesity has surfaced as one of the most challenging worldwide problems that requires urgent attention from the healthcare providers, academics and policy makers. The obesity epidemic is the outcome of a multifaceted intricate interaction between environmental factors, genetic susceptibility, and human behaviour. The effect of genetics and disease on weight gain has been well illustrated in several studies. However, environmental factors are likely to be the main factors driving the rise in the obesity epidemic. It is certain that obesity is due to imbalance between energy intake and energy expenditure. Several published epidemiological studies examined different aspects of the aetiology of Obesity. The ever-growing evidence and continually expanding knowledge have demonstrated the complexity of the issue. Utilising evidence from these studies, this thorough review aims at carrying an in-depth analysis of the different factors contributing to the development of the obesity epidemic.
\end{abstract}

Keywords: obesity, weight gain, health promotion, nutrition, lifestyle, food, genetics, physical activity, public health
Volume 10 Issue 4 - 2020

\section{Tahir Omer}

Northampton General Hospital, University of Chester, UK

Correspondence: Tahir Omer, Northampton General Hospital, University of Chester, UK,

Email tahir.omer@chester.ac.uk

Received: June 21, 2020 | Published: July 03, 2020
Abbreviations: BMI, body mass index; POMC, pro-opiomelanocortin; $C P E$, carboxypeptidase

\section{Introduction}

Obesity is a medical condition defined as disproportionate fat storage in the body that might adversely affect health. ${ }^{1}$ The prevalence of obesity is drastically rising globally, and it is now considered a critical public health issue. According to the World Health Organisation WHO (2016), there are just under 2 billion overweight adults in the world. More than 600 million of them are considered obese. Around $40 \%$ of adults were overweight in 2016 and just under $15 \%$ were obese. The global prevalence of obesity has increased by threefold between 1975 and 2016. If the trend continues to rise, it is estimated that close to one third of the world adult population will be overweight and more than 1 billion will be obese by 2025 . Obesity is measured using body mass index (BMI) which is calculated by dividing the subject's weight in kilograms by their square height in metres. ${ }^{1} \mathrm{~A}$ BMI of 30 or higher indicates obesity and although BMI is a very useful initial assessment method to diagnose obesity, it is not particularly accurate. It does not always reflect the degree of individual's fat storage with great certainty. The WHO recognises this limitation and recommends its use as a screening tool only. Obesity infers a huge economic burden on the already outstretched health systems in many countries. The economic cost of obesity in England has been anticipated at being between $£ 3.3-3.7$ billion with an additional $£ 3-3.7$ billion related cost to treating overweight individuals. ${ }^{2}$

With its sharply rising prevalence, complex aetiology and incapacitating adverse health and economic outcome, Obesity is now considered a global epidemic. Several published epidemiological studies examined different aspects of the complex aetiology of Obesity. Utilising evidence from these studies, this review aims at carrying an in-depth analysis of the different factors contributing to the development of the obesity epidemic.

\section{The causes of obesity}

Obesity ensues when an individual's body accumulates abnormal amounts of fat. This takes place when energy intake exceeds energy expenditure over time. ${ }^{3}$ Many factors could contribute to the rising obesity epidemic.

\section{The role of genetics}

To start with, the importance of genetical predisposition to obesity has been demonstrated in several studies. In a study that examined the effect of increased caloric intake on monozygotic twins in Canada, 12 pairs of identical male twins were provided with extra intake of $1000 \mathrm{kcal}$ per day for six days a week over a period of 100 days. ${ }^{4}$ The overall weight gain was in the range of 4 to $13 \mathrm{~kg}$. Within pairs of twins, the weight gain patterns were almost similar in terms of overall weight, fat distribution and percentage. These similar patterns of response of identical twins clearly highlights the effect of genetical susceptibility on weight. There was greater variation in weight gain in between different twin pairs than within the same pair.

Another study published in the New England Journal of Medicine reviewing 540 adopted adults provides additional evidence of the influence of genetics on body weight..$^{5}$ A group of adults who were adopted as children in Denmark were classified into four groups according to their BMI ranging from underweight to obese. Remarkably, their BMI class was very much related to that of their biological parents $(\mathrm{P}<0.0001$ for mothers and $\mathrm{P}<0.02$ for fathers). There was no significant relationship between the adoptees BMI class and their adopting parents. An additional example of the significance of polygenic susceptibility to weight gain is the Pima Indians of Arizona who are well known as a group to have a high prevalence rate of obesity. This could be related to polygenic aetiology. However, there is considerable variability in the overall response to high calorie intake among them. Weyer et al. ${ }^{6}$ offered that among this group, those 
individuals who can compensate well for the high energy intake by increasing their energy expenditure tend to be less provident in response to deprivation. These adaptive increases and decreases in energy expenditure in response to change in calorie intake play an important role in regulating individual's body weight.

The genetic predisposition to obesity can be polygenic, monogenic or syndromic. ${ }^{7}$ The most well-known monogenic condition causing obesity is the Melanocortin $4 \mathrm{MC} 4$ receptor deficiency due to polymorphism affecting chromosome $18 .{ }^{8}$ Other monogenic causes of obesity include: Pro-opio-melanocortin (POMC) mutations, Proconvertase (PC1/2) deficiency, SIM1 deficiency, NTRK2/ $B D N F$ mutations, $S H 2 B 1$ mutations, kinase suppressor of Ras 2 (KSR2 mutations, mutation in the TUB (tubby-like protein) gene and carboxypeptidase (CPE) gene. ${ }^{7,8}$ Some other rare genetic defects leading to severe childhood obesity have been recognised. These are similar to known genetic defects causing obesity in rats and are secondary to either relative or absolute leptin deficiency and leptin receptor defects. Leptin is an adipocyte driven hormone that acts on the hypothalamus to induce satiety and reduce food intake. Patients with leptin deficiency develop sever obesity secondary to boosted food intake mainly due to lack of satiety and reduced energy expenditure. However, trials of utilising synthetic leptin to treat obesity in humans provided disappointing results ${ }^{10}$ indicating that leptin deficiency is not an important aetiological element in most forms of human obesity. Some other rare genetic conditions can lead to syndromic obesity due to single gene defect or large chromosomal region of multiple genes. This include: Prader-Willi syndrome, Laurence-moon-Beidl syndrome, Cohen syndrome, Albright's hereditary osteodystrophy and Borjeson Forssman Lehmann. However, the exact molecular mechanism that causes weight gain in most of them remains unclear. ${ }^{11}$

\section{Extragenetic causes of obesity}

\section{Weight gain secondary to medical conditions and medications}

The association between several endocrinological conditions and obesity is well recognised. Diseases like Cushing's disease and thyroid underactivity can lead to obesity. Polycystic ovary syndrome leading to insulin resistance may contribute to obesity as well. ${ }^{12}$ Some drugs can also result in rapid weight gain including steroids and antidepressants. ${ }^{13,14}$ Wharton et al..$^{14}$ offer in an extensive review of the effect of different medication classes on weight that some Antipsychotics (including Lithium, Clozapine, Quetiapine and Haloperidol) have greater effect on weight gain that others. $30 \%$ to nearly $90 \%$ of patients receiving clozapine will put on weight and up to one third of patients taking olanzapine will gain more than $5 \%$ of their body weight. Lithium is associated with lesser but significant weight gain of up to $10 \mathrm{~kg}$.

In addition, many antidepressants including Tricyclic antidepressants like Amitriptyline (Average weight gain of 0.5$>7.0 \mathrm{~kg}$ ) and nortriptyline (Average gain of up to $4 \mathrm{~kg}$ ) and MOAIs like Phenelzine and some SSRIs like Citalopram (Average gain of $0.1-7 \mathrm{~kg}$ ) are well associated with weight gain. Glucose lowering medications including Insulin, Sulfonylureas and Thiazolidinediones and some antihypertensives like Clonidine and Atenolol can result in significant weight gain as well.

\section{Individual and socioeconomic factors}

Three main factors contribute to energy expenditure. ${ }^{15}$ The first is the basal metabolic rate (BMR), which is the energy required to maintain normal cellular metabolism at the state of rest. In addition, energy is required for the breaking down of food, and lastly there is the energy required for any physical activity. Both calorie intake and energy expenditure are controlled by the central nervous system. This involves different neurotransmitters and complex mechanisms within the hypothalamus to induce either satiety or hunger. This is guided by genetic predisposition, but it is also subject to voluntary control to some extent. In evolution most of these systems are set to save energy in times of scarcity rather than reducing or wasting it at times of redundancy. ${ }^{15}$ It is clear that whatever role the genetical predisposition plays in weight gain, obesity will only happen if the environment is encouraging such gain (such as energy dense food and inactive lifestyle).${ }^{16}$ The environment has been a significant factor contributing to the sharp rise in obesity prevalence in the last few years. $3,15,16$

The significance of the contribution of environmental factors is shown on a study carried on 2 groups of Pima Indians. Those living in Arizona in the United states have a recognised high rate of obesity. In spite of the similar genetic susceptibility (as they share the same ancestors), Pima Indians living in an isolation in Mexico had considerably lower BMI. The two groups were separated around $500-1000$ years ago and are now totally different in terms of both diet composition/ caloric intake and energy expenditure. The Mexican Pima Indians consume a simpler diet lower in fat and caloric density than their peers in Arizona and their heavy physical work lead to greater levels of energy expenditure. ${ }^{17}$

When considering environmental factors, the fundamental factors that have likely contributed to the obesity epidemics are the changes to the amount and quality of food in the diet (energy density) and the changes in the level of energy expenditure in terms of physical activity carried out by the population. The redundancy and easy accessibility to a variety of high caloric diets, rich in fat and processed carbohydrate is a crucial factor in the obesity epidemic. ${ }^{18}$ Soft drinks, alcoholic beverages and fast food tend to be calorie rich.

In Britain, there has been a significant rise in the amount of fat in diet by more than $50 \%$ over the past 50 years. ${ }^{19}$ On the other hand, levels of energy expenditure in terms of physical activity have decreased significantly in the same period with less manual jobs and the wide utilisation of cars as a mode of transport. Car driving and time spent on TV viewing significantly correlate with the rise in obesity in England. TV viewing time in specific is a growing concern amongst children as a contributor to the rise in childhood obesity prevalence. ${ }^{19}$ In addition, evidence suggests that the affordability and relative obtainability of different food products clearly affect food consumption. ${ }^{20}$

The point of purchase effect has a major role in the obesity epidemic. ${ }^{21}$ In-store environment is when people are likely to make unwise and almost impulsive decisions about the type and amount of food to buy. People are often unable to recognize and avoid purchasing a product due to the complex marketing strategies that sometimes work through non-cognitive processes. The food industry recognises the role of product presentation in influencing purchasing. In fact, this was acknowledged in the 1970s and since then worldwide, the food processing industry has been spending more into in-store marketing. In the US, food industry expenditure on in-store advertising was just above $25 \%$ of the marketing cost in the 1960 s. This has jumped up to close to $70 \%$ in $2009 .^{21}$

Obesity, although mainly seen in the economically privileged western countries, is more prevalent in subjects from lower socio- 
economic classes. This is likely due to the high fat content in diet in these groups and reduced physical activity especially with the regression in customary manual occupations. A systemic review carried out in $2007^{22}$ showed that ethnic minority and lowsocioeconomic-status groups in the United States are more affected by obesity at all ages. In 2003, ethnic minority groups had a higher prevalence of obesity than whites by almost $10 \%$ (76.1 percent versus 64.2 percent). These differences where recognised more among males than females.

Interestingly, another systemic review of different studies examining the association between the socioeconomic class and obesity in developing countries ${ }^{23}$ showed that in low-income countries, Individuals with higher income and/or higher level of education are more likely to be overweight. In developing countries with higher income, the previously noted correlation becomes mixed for men and nearly non-existent for women. Childhood obesity seems to mostly affect the higher socioeconomic class in developing countries.

A clear likely explanation for that, would be food scarcity in low-income countries which leads to reduced food intake among the unfortunate families with low income. In addition, those individuals are likely to be very physically active engaging in labour manual work. Conversely, the rich are more prone to obesity due to accessibility to excess food and less involvement in manual occupations. It is also common in many cultures to consider obesity and overweight as a sign of affluency. By contrast, in developing countries with higher income, food scarcity becomes less of an issue. In its place, access to healthy food becomes the driving factor differentiating the more from the less affluent. Healthy food with low energy density (e.g. wholegrain cereals, fruits and vegetables) will likely be unaffordable for the low socioeconomic group, thus leading to the consumption of an affordable less healthy and more energy dense diet as shown on a study from 2011 in rural South Africa which compared the prices of some of the common consumed food and reported that healthier diets cost up to $60 \%$ more than the less healthy options. ${ }^{24}$

\section{Environmental factors}

The effect of workplace environment on the prevalence obesity is well recognised. Long working hours can result in increased BMI due to reduced time for exercise and physical activity. It can also result in shifting towards ready processed meals and fast food rather than home-made meals. This could also influence the individual's children by reducing supervision time. This was examined in a study in $2009^{25}$ which reported significant association between longer working hours and increased BMI of the individual and their children, albeit insignificant increment in the BMI of the subject's partner $(10 \mathrm{hr}$ work increases $\mathrm{P}$ by a statistically significant 1.3 percentage points and has essentially no effect on partner's P). Another observational study that reviewed more than 1100 participants ${ }^{26}$ reported increased BMI with long working hours in women (ORs for obesity attributable to long working hours were 2.42 (95\% CI, 1.05-5.57)).

Long working hours can lead to reduced sleeping hours and contribute to obesity. A cross sectional observational study in Hong Kong including more than 4700 participants with around 49\% Male and $51 \%$ female subjects showed increased number of working hours and less sleeping hours were significantly associated with increased BMI. ${ }^{27}$ Subjects who worked longer than 9 hours had the highest BMI and waist circumference $(\mathrm{r}=0.084, \mathrm{Po} 0.001)$. However, the questionnaire utilised in the study was broadly general and there was lack of assessment of reliability creating measurement bias. Nevertheless, this study reaffirmed the speculated association between longer working hours, lack of sleep and obesity.

Lack of sleep can result in hormonal imbalance (impaired glucose tolerance and increased nocturnal cortisol) and adversely affect the cognitive function leading to obesity. ${ }^{28} \mathrm{~A}$ prospective study recruited more than 100 patients from primary care in the USA to review the association between sleeping hours and BMI. ${ }^{28}$ There was a linear relationship between lack of sleep and increased BMI. Among the strengths of this. study is the large heterogenous groups of subjects from different sites. However, due to the observational nature of the study, confounding factors cannot be ruled out.

The inverse association between sleeping hours and obesity was demonstrated in several studies globally. More than 8000 children in Japan were reviewed in a study in $2002 .{ }^{29}$ Significant association between sleeping hours and BMI was reported among the children with adjusted odds ratio of 1.49 (95\% confidence interval 1.08-2.14) for more than 9 hours sleep, 1.89 (1.34-2.73) for 8 to 9 hours and 2.87 (1.61-5.05) for less than 8 hours of sleep. Similarly, another study involving 300 subjects demonstrated increased risk of obesity with less than 6 hours of sleep or sleeping beyond midnight. ${ }^{30}$ In Australia, more than 1100 participants were reviewed in a crosssectional population ${ }^{31}$ to examine the relationship between working conditions and obesity. They noted that longer hours of work are associated with increased BMI. Interestingly, there was no association between job strain and BMI, however high psychological demand and low reward contributed to raised BMI.

In addition, the built environment can influence travel and commuting choices in a financial and behavioural perspectives. ${ }^{32}$ Vehicle trips are linked to several adverse outcomes. Households would make less vehicle trips if located in areas with denser land use development and better transit service. In other words, in order to reduce vehicle use, planning neighbourhoods should focus on high land use developments to shorten the distance to transit and make destinations more accessible by providing easy access to trip attractions. A systemic review ${ }^{33}$ on the relationship between the built environment and obesity showed that access to physical activity opportunities and access to food outlet can significantly affect body weight. Lack of sidewalks and poor access to recreational facilities was associated with obesity/ overweight. While increased mixed land use and daily distance walked were associated with reduced obesity, increased time spent in a car was associated with the reverse. Increased open spaces and local parks availability was associated with reduced obesity.

There was positive association between high BMI and vehicle miles travelled $(\mathrm{r}=0.79)$ and commute time $(\mathrm{r}=0.55)$ and a negative correlation between high BMI and population density ( $\mathrm{r}=-0.342)$ (all $p$ 's $<0.05$ ).

Finally, the role of social networks and peer effect in the rise of obesity prevalence cannot be ignored. This was examined in a study that reviewed a social network of more than 12,000 people from the Framingham Heart Study to review the influence of weight gain on an individual's network of friends, partner and family. ${ }^{34}$ Interestingly, an Individual's risk of weight gain increases by more than $50 \%$ if a friend became obese and by more than $30 \%$ if a partner is obese. The determinants of obesity are summarised in Table 1 . 
Table I Summary of the risk factors and contributors to obesity

Genetics: Polygenic, monogenic or syndromic, Extragenetic causes

\section{Individual:}

Certain endocrinological conditions: Cushing's disease, PCOS, Hypothyroidism.

Certain medications:Anti-psychotics, Anti-depressants, Anti-Diabetic, Antihypertensives, and steroids.

Depression, Anxiety, stress.

Energy intake exceeding energy expenditure due to excessive intake of energy-dense food and reduced physical activity.

Health inequalities and socioeconomic factors: Low income and low educational level.

Sleep deprivation.

Environmental:

The workplace environment and long working hours.

The built environment: poor access to recreational facilities, lack of sidewalks, Food deserts due to lack of food outlets.

Social networks.

\section{Conclusion}

The sharp rise in Obesity prevalence has raised major global concern. Clearly, the obesity epidemic is the outcome of multifaceted interaction between the environmental factors, genetic susceptibility, and human behaviour. The effect of genetics and disease on weight gain has been well illustrated in several studies. However, environmental factors are likely to be the main factors driving the rise in the obesity epidemic. It is certain that obesity is due to imbalance between energy intake and energy expenditure. Excess energy dense food intake and reduced physical activity are likely the major contributors to obesity. There is also evidence that the readily available and affordability of different food products affect food consumption. Low socioeconomic status, the peer effect and social networks are well known risk factors to the development of obesity. The obesity epidemic deploys a detrimental impact on the economy with its high healthcare cost. ${ }^{35}$ The problem of overweight and obesity has therefore surfaced as one of the most challenging worldwide problems over the coming decades, and requires urgent attention from the healthcare providers, policy makers, and academics.

\section{Acknowledgments}

Author would like to thank all the contributors to the field of obesity for their continuous effort in demonstrating the causes, consequences and possible solutions to this complex condition.

\section{Conflicts of interest}

There are no conflicts of interest.

\section{Funding}

None.

\section{References}

1. World Health Organization. Fact sheet: obesity and overweight. 2019.

2. The house of commons health committee report. 2004.

3. Wilding J. Are the causes of obesity primary environmental? Yes. BMJ 2012;11(34):e5843.
4. Bouchard C, Tremblay A, Després JP. The response to long-term overfeeding in identical twins. N Engl J Med. 1990;322:477-1482.

5. Stunkard AJ, Sorensen TI, Hanis C, et al. An adoption study of human obesity. N Engl J MeD. 1986;314(4):193-198.

6. Weyer C, Vozarova B, Ravussin E, et al. Changes in energy metabolism in response to $48 \mathrm{~h}$ of overfeeding and fasting in Caucasians and Pima Indians. Int J Obesity. 2001;25:593-600.

7. Thaker VV. Genetic and Epigenetic causes of Obesity. Adolescent medicine: state of the art reviews. 2017;28(2):379-405.

8. Adan R, Tiesjema B, Hillebrand J, et al. The MC4 receptor and control of appetite. British Journal of Pharmacology. 2006;149(7):815-827.

9. Montague CT, Farooqi IS, Whitehead JP, et al. Congenital leptin deficiency is associated with severe early-onset obesity in humans. Nature. 1997;387(6636):903-908.

10. Heymsfield SB, Greenberg AS, Fujioka K, et al. Recombinant leptin for weight loss in obese and lean adults - a randomized, controlled, doseescalation trial. JAMA. 1999;282(16):1568-1575.

11. Webb T, Clarke D, Hardy CA, et al. Clinical, cytogenetic, and molecular study of 40 adults with the Prader-Willi syndrome. J Med Genet. 1995;32:181-185.

12. Legro RS. Obesity and PCOS: implications for diagnosis and treatment. Seminars in reproductive medicine. 2012;30(6):496-506.

13. Kopleman PG, Caterson ID, Dietz WH. Clinical Obesity in Adults and Children. 3rd ed. London, UK: Wiley-Blackwell; 2010.

14. Wharton S, Raiber L, Serodio K, et al. Medications that cause weight gain and alternatives in Canada: a narrative review', Diabetes, Metabolic Syndrome and Obesity: Targets and Therapy. 2018;11:427-438.

15. Wilding JPH. Causes of obesity' Practical Diabetes. 2001;18(4):288-299.

16. Stunkard A, Sørensen T, Hanis C, et al. An Adoption Study of Human Obesity. New England Journal of Medicine. 1986;314(4):193-198.

17. Ravussin E, Valencia ME, Esparza J, et al. Effects of a traditional lifestyle on obesity in Pima Indians. Diabetes Care. 1994;17(9):1067-1074.

18. Nguyen D, El-Serag H. The Epidemiology of Obesity. Gastroenterology Clinics of North America. 2010;39(1):1-7. 
19. Prentice AM, Jebb SA. Obesity in Britain: gluttony or sloth? BMJ. 1995;311(7002):437-439.

20. Holsten JE. Obesity and the community food environment: a systematic review. Public Health Nutr. 2008;20(14):1-9.

21. Cohen DA, Lesser LI. Obesity prevention at the point of purchase. Obesity reviews. 2016;17(5):389-396.

22. Wang Y, Beydoun MA. The obesity epidemic in the United Statesgender, age, socioeconomic, racial/ethnic, and geographic characteristics: a systematic review and meta-regression analysis. Epidemiologic Reviews. 2007;29(1):6-19.

23. Dinsa G, Goryakin Y, Fumagalli E, et al. Obesity and socioeconomic status in developing countries: a systematic review. Obesity Reviews. 2012;13(11):1067-1079.

24. Temple NJ, Steyn NP, Fourie J, et al. Price and availability of healthy food: a study in rural South Africa. Nutrition. 2011;27(1):5558.

25. Courtemanche C. Longer Hours and Larger Waistlines? The Relationship Between Work Hours and Obesity. SSRN Electronic Journal. 2009.

26. Kim B, Lee B, Park H, et al. Long working hours and overweight and obesity in working adults. Annals of Occupational and Environmental Medicine. 2016;28(1).

27. Ko G, Chan J, Chan A, et al. Association between sleeping hours, working hours and obesity in Hong Kong Chinese: the 'better health for better Hong Kong' health promotion campaign. International Journal of Obesity. 2006;31(2):254-260.
28. Vorona R, Winn M, Babineau T, et al. Overweight and Obese Patients in a Primary Care Population Report Less Sleep Than Patients With a Normal Body Mass Index. Archives of Internal Medicine. 2005;165(1):25.

29. Sekine M, Yamagami T, Handa K, et al. A dose-response relationship between short sleeping hours and childhood obesity: results of the Toyama Birth Cohort Study. Child: Care, Health and Development. 2002;28(2):163170.

30. Shigeta H, Shigeta M, Nakazawa A, et al. Lifestyle, Obesity, and Insulin Resistance. Diabetes Care. 2001;24(3):608-608.

31. Ostry A, Radi S, Louie A et al. Psychosocial and other working conditions in relation to body mass index in a representative sample of Australian workers. BMC Public Health. 2006;6(1).

32. Zhang Y, Wu W, Li Y, et al. Does the Built Environment Make a Difference? An Investigation of Household Vehicle Use in Zhongshan Metropolitan Area, China. Sustainability. 2014;6(8):4910-4930.

33. Papas M, Alberg A, Ewing R, et al. The Built Environment and Obesity. Epidemiologic Reviews. 2007;29(1):129-143.

34. Christakis NA, Fowler JH.The spread of obesity in a large social network over 32 years. $N$ Engl J Med. 2007;357(4):370-379.

35. Withrow D, Alter DA. The economic burden of obesity worldwide: a systematic review of the direct costs of obesity. Obesity reviews. 2011;12(2):131-141. 